\title{
Viscosity approximation methods for nonexpansive nonself-mappings without boundary conditions
}

\author{
Haiyun Zhou ${ }^{1,2^{*}}$ and Peiyuan Wang ${ }^{2}$
}

\author{
"Correspondence: \\ zhouhy123@aliyun.com \\ 'Department of Mathematics and \\ Information, Hebei Normal \\ University, Shijiazhuang, 050024, \\ China \\ ${ }^{2}$ Department of Mathematics, \\ Shijiazhuang Mechanical \\ Engineering College, Shijiazhuang, \\ 050003, China
}

\begin{abstract}
In this paper, we introduce several types of viscosity approximation methods for nonexpansive nonself-mappings in certain Banach spaces. Using new analysis techniques, we prove several strong convergence theorems for nonexpansive nonself-mappings in certain Banach spaces without boundary conditions.

MSC: $47 \mathrm{H} 05 ; 47 \mathrm{H} 09 ; 47 \mathrm{H} 10$
\end{abstract}

Keywords: nonexpansive nonself-mapping; sunny nonexpansive retraction; boundary condition; fixed point; viscosity approximation

\section{Introduction}

Let $C$ be a nonempty closed convex subset of a real Banach space $E$ and let $T$ be a nonexpansive mapping defined on $C$, that is, $\|T x-T y\| \leq\|x-y\|$ for all $x, y \in C$. Denote by $\operatorname{Fix}(T)$ the set of fixed points of $T$; that is, $\operatorname{Fix}(T)=\{x \in C: T x=x\}$.

In general, the domain of $T$ is a proper subset of $E$. In this case, some iterative algorithms associated with $T$ may not be well defined. Therefore, it is interesting to find some conditions under which the algorithms considered are well defined.

Recently, the problem of approximating fixed points for nonexpansive nonself-mappings has been paid much attention to by many authors, see [1-4]. Moreover, in [5], Song and Chen introduced two iterative methods (one is implicit and the other is explicit) and established the strong convergence of such two methods in certain Banach spaces, if $T$ satisfies the weakly inward condition and $\operatorname{Fix}(T) \neq \emptyset$. Further, in [6], Matsushita and Takahashi introduced a new condition (2.2). They proved the following. (i) If $T$ satisfies the weakly inward condition, then $T$ satisfies the condition (2.2). (ii) If $E$ is a strictly convex Banach space, $T: C \rightarrow E$ is a nonexpansive mapping such that $\operatorname{Fix}(T) \neq \emptyset$ and $C$ is a sunny nonexpansive retract of $E$, then $T$ satisfies the condition (2.2). (iii) If $T$ satisfies the condition (2.2), then $\operatorname{Fix}(T)=\operatorname{Fix}(Q T)$, where $Q$ is a sunny nonexpansive retraction from $E$ onto $C$. Using these results, they proved two strong convergence theorems for nonexpansive nonself-mappings in certain Banach spaces without any boundary conditions.

We remark in the passing that the convergence theorems of Song and Chen [5] are not applicable to $l^{p}$ and $L^{p}$ for any $p \in(0,2) \cup(2, \infty)$. Although the convergence theorems of Matsushita and Takahashi [6] work in the Banach spaces of $l^{p}$ and $L^{p}$ for all $p>1$, they could not be used to find the minimum-norm fixed point of the underlying mappings in a Hilbert space. Moreover, the proof lines of Theorem 4.2 in [6] are really long.

(O2014 Zhou and Wang; licensee Springer. This is an Open Access article distributed under the terms of the Creative Commons Attribution License (http://creativecommons.org/licenses/by/2.0), which permits unrestricted use, distribution, and reproduction in any medium, provided the original work is properly cited. 
Our concern now is the following.

(1) Can one present an indirect and simple method for proving Theorem 4.2 of Matsushita and Takahashi [6]?

(2) Can one extend Theorem 4.2 to both the more general Banach spaces and the more broad Meir-Keeler contractions?

(3) Can one improve the main results of [6] so that new convergence theorems hold true under less assumptions?

The purpose of this paper is to study and solve all problems mentioned above by improving and generalizing several recent results. Several new iterative schemes are introduced and several strong convergence theorems are established by using new analysis techniques.

\section{Preliminaries}

Let $E$ be a real Banach space and let $E^{*}$ be the dual of $E$. Denote by $\langle\cdot, \cdot\rangle$ the duality product. A Banach space $E$ is said to be strictly convex if $\left\|\frac{x+y}{2}\right\|<1$ for all $x, y \in E$ with $\|x\|=\|y\|=$ 1 and $x \neq y$. It is also said to be uniformly convex if $\lim _{x \rightarrow \infty}\left\|x_{n}-y_{n}\right\|=0$ for any two sequences $\left\{x_{n}\right\},\left\{y_{n}\right\}$ in $E$ such that $\left\|x_{n}\right\|=\left\|y_{n}\right\|=1$ and $\lim _{x \rightarrow \infty}\left\|x_{n}+y_{n}\right\|=2$. Let $U=\{x \in$ $E:\|x\|=1$ \} be the unit sphere of $E$. The norm of $E$ is said to be Gâteaux differentiable if the limit

$$
\lim _{t \rightarrow 0} \frac{\|x+t y\|-\|x\|}{t}
$$

exists for each $x, y \in U$. Such an $E$ is called a smooth Banach space. The norm of a Banach space $E$ is said to be uniformly Gâteaux differentiable if for each $y$ in $U$, the limit of (2.1) is attained uniformly for $x$ in $U$. The norm of $E$ is said to be Fréchet differentiable if for each $x \in U$, the limit of (2.1) is attained uniformly for $y \in U$. The norm of $E$ is also said to be uniformly Fréchet differentiable if the limit of (2.1) is attained uniformly for $x, y \in U$. In this case, $E$ is called a uniformly smooth Banach space. The normalized duality mapping $J$ from $E$ into $2^{E^{*}}$ is defined by

$$
J x=\left\{x^{*} \in E^{*}:\left\langle x, x^{*}\right\rangle=\|x\|^{2}=\left\|x^{*}\right\|\right\}
$$

for each $x \in E$. The duality mapping $J$ has the following basic properties; see [7, 8] for details.

\section{Proposition 2.1}

(1) $\|x\|^{2}-\|y\|^{2} \geq 2\langle x-y, j\rangle$ for each $x, y \in E$ and $j \in J y$.

(2) If $E$ is smooth, then $J$ is single valued.

(3) If $E$ is smooth, then $J$ is norm-to-weak* continuous.

(4) If E has a uniformly Gâteaux differentiable norm, then J is norm-to-weak* uniformly continuous on bounded sets of $E$.

(5) If the norm of $E$ is (uniformly) Fréchet differentiable, then it is (uniformly) Gâteaux differentiable.

(6) If Banach space $E$ is uniformly smooth, then J is norm-to-norm uniformly continuous on bounded sets of $E$. 
Recall that a mapping $T: C \rightarrow E$ is said to satisfy the weakly inward condition $[7,8]$ if $T x \in \operatorname{cl} I_{C}(x)$ for all $x \in C$, where

$$
I_{C}(x)=\{y \in E: y=x+a(u-x), u \in C, a \geq 0\}
$$

and $\operatorname{cl} I_{C}(x)$ denotes the closure of the set $I_{C}(x)$.

Remark 2.1 (i) $C \subset I_{C}(x)$ for all $x \in C$;

(ii) if $x$ is an interior of $C$, then $I_{C}(x)=E$;

(iii) if $C$ is a convex subset of $E$, then $I_{C}(x)$ is also convex and

$$
I_{C}(x)=\{y \in E: y=x+a(u-x), u \in C, a \geq 1\}
$$

(iv) if both $f: C \rightarrow E$ and $g: C \rightarrow E$ satisfy the weakly inward condition, then so does a convex combination of $f$ and $g$.

Let $D$ be a subset of $C$ and let $Q$ be a mapping from $C$ to $D$. Then $Q$ is said to be sunny if $Q(Q x+t(x-Q x))=Q x$ whenever $Q x+t(x-Q x) \in C$ for $x \in C$ and $t \geq 0$. A mapping $Q$ from $C$ into itself is said to be a retraction if $Q^{2}=Q$. A set $D$ is said to be a sunny nonexpansive retract of $C$ if there exists a sunny nonexpansive retraction from $C$ into $D[7,8]$. It is very well known that if $E$ is a smooth Banach space and $C$ is a nonempty closed convex subset of $E$, then there exists at most one sunny nonexpansive retraction $Q$ from $E$ onto $C$. In a Hilbert space $H$, there exists a unique sunny nonexpansive retraction $P_{C}: H \rightarrow C$.

Proposition 2.2 Let $C$ be a nonempty convex subset of a real smooth Banach space $E$, $D \subset C$ and $Q: C \rightarrow D$ a retraction. Then the following statements are equivalent:

(1) $Q$ is both sunny and nonexpansive;

(2) $\langle x-Q x, j(y-Q x)\rangle \leq 0$ for all $x \in C$ and $y \in D$;

(3) $\langle x-y, j(Q x-Q y)\rangle \geq\|Q x-Q y\|^{2}$ for all $x, y \in C$.

Let $Q$ be a sunny nonexpansive retraction from $E$ to $C$. Define a set

$$
S_{x}=\{y \in E: y \neq x, Q y=x\}
$$

for $x \in C$. A nonself-mapping $T: C \rightarrow E$ is said to satisfy the Matsushita-Takahashi condition (MT condition, for short) if

$$
T x \in S_{x}^{C}
$$

for all $x \in C$, where $S_{x}^{C}$ denotes the complementary set of $S_{x}$. If $E$ is a Hilbert space, then the MT condition is equivalent to the nowhere-normal outward condition introduced by Matsushita and Kuroiwa [9].

Matsushita and Takahashi [6] proved the following interesting results.

Proposition 2.3 Let $C$ be a closed convex subset of a smooth Banach space $E$ and let $T$ be a mapping from $C$ into $E$. Suppose that $C$ is a sunny nonexpansive retract of $E$. If $T$ satisfies the weakly inward condition, then $T$ satisfies the MT condition. 
Proposition 2.4 Let $C$ be a closed convex subset of a strictly convex Banach space $E$ and let $T$ be a mapping from $C$ into $E$. Suppose that $C$ is a sunny nonexpansive retract of $E$. If $\operatorname{Fix}(T) \neq \emptyset$, then $T$ satisfies the MT condition.

Proposition 2.5 Let $C$ be a closed convex subset of a smooth Banach space $E$ and let $T$ be a mapping form $C$ into $E$. Suppose that $C$ is a sunny nonexpansive retract of $E$. If $T$ satisfies the MT condition, then

$$
\operatorname{Fix}(T)=\operatorname{Fix}(Q T),
$$

where $Q$ is the unique sunny nonexpansive retraction from $E$ onto $C$.

By a careful observation, we have the following more rich and nice result.

Lemma 2.1 Let $C$ be a closed convex subset of smooth Banach space $E$ and let $T$ be a mapping form $C$ into $E$. Suppose that $C$ is a sunny nonexpansive retract of $E$. If $T$ satisfies the MT condition, then

$$
\operatorname{Fix}(T)=\operatorname{Fix}(Q T)=\operatorname{Fix}(T Q),
$$

where $Q$ is the unique sunny nonexpansive retraction form $E$ onto $C$.

Proof The first '=' follows from Proposition 2.5. Now we prove that second '=' holds. Assume that $x=T Q x$; then

$$
Q x=(Q T)(Q x),
$$

which shows that $Q x \in \operatorname{Fix}(Q T)$. In view of Proposition 2.5, $\operatorname{Fix}(Q T)=\operatorname{Fix}(T)$, thus, we get $x=T Q x=Q x$, which turns out that $x=T x$. The converse inclusion relation that ${ }^{\prime} F i x(T) \subset$ $\operatorname{Fix}(T Q)$ ' is obvious, consequently, $\operatorname{Fix}(T)=\operatorname{Fix}(T Q)$. The proof is completed.

Remark 2.2 The fact that $\operatorname{Fix}(T)=\operatorname{Fix}(T Q)$ is very important. By virtue of this fact, we can invent and create some novel argument methods for strong convergence of the some kinds of iterative algorithms.

Let $(X, d)$ be a metric space and $C$ a subset of $X$. Recall that a mapping $\Phi: C \rightarrow C$ is called a Meir-Keeler contraction (MKC for short) [10], if for arbitrary $\varepsilon>0$, there exists $\delta>0$ such that

$$
d(x, y)<\varepsilon+\delta \Rightarrow d(\Phi x, \Phi y)<\varepsilon .
$$

In [10], Meir and Keeler proved the following interesting fixed point theorem.

Theorem MK Let $(X, d)$ be a complete metric space and let $\Phi$ be a MKC on X. Then $\Phi$ has a unique fixed point in $X$.

Remark 2.3 If $C$ is a nonempty closed (convex) subset of a complete metric space $(X, d)$, then the conclusion of Theorem MK is still true. 
The following results can be found in [11].

Proposition 2.6 Let $E$ be a Banach space $C$ a convex subset of $E$, and $\Phi: C \rightarrow C$ a $M K C$. Then $\forall \varepsilon>0$, there exists $\gamma \in(0,1)$ such that

$$
\|\Phi(x)-\Phi(y)\| \leq \gamma\|x-y\|
$$

for all $x, y \in C$ with $\|x-y\| \geq \varepsilon$.

Proposition 2.7 Let $C$ be a nonempty convex subset of a Banach space $E, T: C \rightarrow C$ a nonexpansive mapping and $\Phi: C \rightarrow C$ a MKC. Then

(i) $T \Phi$ and $\Phi T: C \rightarrow C$ are all Meir-Keeler contractions;

(ii) $\forall t \in(0,1)$, define a mapping $T_{t}: C \rightarrow C$ by

$$
T_{t}(x)=(1-t) T x+t \Phi x, \quad x \in C,
$$

then $T_{t}$ is a $M K C$.

Remark 2.4 When $\Phi$ is not a self-mapping, then conclusion of (i) is still true, provided that $T \Phi$ or $\Phi T$ is well defined. The conclusion of (ii) holds true for nonself-mappings $T$ and $\Phi$

Proposition 2.8 Let $C$ be a nonempty closed convex subset of a reflexive and strictly convex Banach space $E$, whose norm is uniformly Gâteaux differentiable. Let $T: C \rightarrow C$ be a nonexpansive mapping with $\operatorname{Fix}(T)=\varnothing$ and $\Phi: C \rightarrow C$ a MKC. Then the following statements are true:

(i) $\forall t \in(0,1]$, there exists a unique continuous path $\left\{x_{t}\right\}$ such that

$$
x_{t}=t \Phi x_{t}+(1-t) T x_{t}, \quad t \in(0,1],
$$

further, as $t \rightarrow 0,\left\{x_{t}\right\}$ converges strongly to a fixed point $z$ of $T$, which solves the following variational inequality:

$$
\text { (VI) }\langle(I-\Phi) z, j(y-z)\rangle \geq 0, \quad \forall y \in \operatorname{Fix}(T),
$$

(ii) for arbitrary initial data $x_{1} \in C$, a sequence $\left\{x_{n}\right\}$ is generated by

$$
x_{n+1}=\alpha_{n} \Phi x_{n}+\left(1-\alpha_{n}\right) T x_{n}, \quad n \geq 1,
$$

where $\left\{\alpha_{n}\right\}$ is a real sequence satisfying conditions:

$$
\begin{aligned}
& (\mathrm{C} 1) \alpha_{n} \rightarrow 0 \text {; (C2) } \sum_{n=1}^{\infty} \alpha_{n}=\infty \text {; and (C3) } \sum_{n=1}^{\infty}\left|\alpha_{n+1}-\alpha_{n}\right|<\infty \text { or } \frac{\alpha_{n}}{\alpha_{n+1}} \rightarrow 1 \\
& (n \rightarrow \infty) .
\end{aligned}
$$

Then $\left\{x_{n}\right\}$ converges strongly to the same point $z$ as in (i).

Let $C$ be a nonempty closed convex subset of a Banach space $E, \Phi: C \rightarrow E$ a MKC and $T: C \rightarrow E$ a nonexpansive mapping. Let $Q$ be a sunny nonexpansive retraction form $E$ onto $C$. For each $t \in(0,1)$, the mapping $t \Phi+(1-t) T$ is a MKC by Proposition 2.7(ii), and 
hence the mapping $Q[t \Phi+(1-t) T]$ is a MKC by Proposition 2.7(i). Then the fixed point Theorem MK ensures that for each $t \in(0,1)$, there exists a unique element $x_{t} \in C$ such that

$$
x_{t}=Q\left[t \Phi\left(x_{t}\right)+(1-t) T\left(x_{t}\right)\right]
$$

which inspires us to consider the following discrete iterative sequence $\left\{x_{n}\right\}$ :

$$
x_{1} \in C, \quad x_{n+1}=Q\left[\alpha_{n} \Phi\left(x_{n}\right)+\left(1-\alpha_{n}\right) T x_{n}\right], \quad n \geq 1,
$$

where $\left\{\alpha_{n}\right\}$ is a real sequence that satisfies certain conditions.

We also consider a variant of (2.8):

$$
\lambda \in(0,1), x_{1} \in C, \quad x_{n+1}=\lambda x_{n}+(1-\lambda) Q\left[\alpha_{n} \Phi\left(x_{n}\right)+\left(1-\alpha_{n}\right) T x_{n}\right], \quad n \geq 1,
$$

where $\left\{\alpha_{n}\right\}$ is a real sequence that satisfies less restriction. We shall prove that the path defined by (2.7) and the sequences defined by (2.8) and (2.9), respectively, converge strongly to a fixed point of $T$ which solves variational inequality (VI).

In order to achieve the objective above, we cite the following known results; see [11].

Proposition 2.9 Let E be a Banach space, $\left\{x_{n}\right\}$ and $\left\{y_{n}\right\}$ two bounded sequences in $E$ that satisfy the relation:

$$
x_{n+1}=\lambda_{n} x_{n}+\left(1-\lambda_{n}\right) y_{n}, \quad n \geq 1,
$$

where $\left\{\lambda_{n}\right\}$ is a real sequence satisfying $0<\underline{\lim }_{n} \lambda_{n} \leq \varlimsup_{n} \lambda_{n}<1$. Then $x_{n}-y_{n} \rightarrow 0(n \rightarrow$ $\infty)$.

Proposition 2.10 Let $\left\{a_{n}\right\}$ be a nonnegative real sequence that satisfies inequality:

$$
a_{n+1} \leq\left(1-t_{n}\right) a_{n}+o\left(t_{n}\right), \quad n \geq 1,
$$

where $\left\{t_{n}\right\}$ is a real sequence in $(0,1)$ satisfying conditions (i) $t_{n} \rightarrow 0$ and (ii) $\sum_{n=1}^{\infty} t_{n}=\infty$. Then $a_{n} \rightarrow 0(n \rightarrow \infty)$.

\section{Main results}

Theorem 3.1 Let $C$ be a nonempty closed convex subset of a reflexive and strictly convex Banach space $E$ whose norm is uniformly Gâteaux differentiable. Let $T: C \rightarrow E$ be a nonexpansive mapping with $\operatorname{Fix}(T) \neq \emptyset$ and $\Phi: C \rightarrow E$ a MKC. Suppose also that $C$ is a sunny nonexpansive retract of $E$ and let $Q$ be the unique sunny nonexpansive retraction from $E$ onto $C$. Then the path $\left\{x_{t}\right\}$ defined by (2.7) converges strongly to $z$ which solves the variational inequality

(VI) $\langle(I-\Phi) z, j(y-z)| \geq 0, \quad \forall y \in \operatorname{Fix}(T)$,

equivalently, $z=Q_{\mathrm{Fix}(T)} \Phi(z)$, where $Q_{\mathrm{Fix}(T)}: E \rightarrow \operatorname{Fix}(T)$ is the unique sunny nonexpansive retraction from $E$ onto $\operatorname{Fix}(T)$. 
Proof Set $y_{t}=t \Phi\left(x_{t}\right)+(1-t) T\left(x_{t}\right)$. Then (2.7) reduces to $x_{t}=Q y_{t}$ and

$$
y_{t}=t(\Phi Q) y_{t}+(1-t)(T Q) y_{t}
$$

Write $f=\Phi Q$ and $V=T Q$. Then (3.1) reduces to

$$
y_{t}=t f\left(y_{t}\right)+(1-t) V\left(y_{t}\right)
$$

In view of Proposition 2.7(i), we know that $f: E \rightarrow E$ is a MKC on $E$ and $V: E \rightarrow E$ is a nonexpansive mapping on $E$. From Proposition 2.8(i), we conclude that $\left\{y_{t}\right\}$ converges strongly to a fixed point $z$ of $(T Q)$, which solves the variational inequality:

$$
\langle(I-f) z, j(y-z)\rangle \geq 0, \quad \forall y \in \operatorname{Fix}(T Q) .
$$

It follows from Proposition 2.4 that $T$ satisfies the MT condition.

By virtue of Lemma 2.1, we have $\operatorname{Fix}(T Q)=\operatorname{Fix}(T)$, which implies that $z \in C$ and hence $Q z=z$, which gives

$$
f(z)=\Phi Q(z)=\Phi(z)
$$

Consequently, $y_{t} \rightarrow z \in \operatorname{Fix}(T)$, and $x_{t} \rightarrow z$ which solves the variational inequality (VI)

$$
\langle(I-\Phi) z, j(y-z)\rangle \geq 0, \quad \forall y \in \operatorname{Fix}(T)
$$

which is equivalent to $z=Q_{\mathrm{Fix}(T)} \Phi(z)$ by Proposition 2.2 .

Theorem 3.2 Let $C$ be a nonempty closed convex subset of a reflexive and strictly convex Banach space E, whose norm is uniformly Gâteaux differentiable. Let $T: C \rightarrow E$ be a nonexpansive mapping with $\operatorname{Fix}(T) \neq \varnothing$ and $\Phi: C \rightarrow E$ a MKC. Suppose also that $C$ is a sunny nonexpansive retract of $E$ and let $Q$ be the unique sunny nonexpansive retraction from $E$ onto $C$. Let $\left\{\alpha_{n}\right\}$ be a real sequence satisfying conditions (C1), (C2), and (C3) given in Proposition 2.8. Then the sequence $\left\{x_{n}\right\}$ defined by (2.8) converges strongly to $z$ which solves the variational inequality (VI)

$$
\langle(I-\Phi) z, j(y-z)\rangle \geq 0, \quad \forall y \in \operatorname{Fix}(T)
$$

Proof Set $y_{n}=\alpha_{n} \Phi\left(x_{n}\right)+\left(1-\alpha_{n}\right) T\left(x_{n}\right)$. Then (2.8) reduces to $x_{n+1}=Q y_{n}$ and

$$
y_{n+1}=\alpha_{n+1}(\Phi Q) y_{n}+\left(1-\alpha_{n+1}\right)(T Q) y_{n} .
$$

Write $\beta_{n}=\alpha_{n+1}, g=\Phi Q$ and $U=T Q$. Then (3.5) reduces to

$$
y_{n+1}=\beta_{n} g\left(y_{n}\right)+\left(1-\beta_{n}\right) U\left(y_{n}\right) .
$$

In view of Proposition 2.7(i), $g: E \rightarrow E$ is a $\mathrm{MKC}$ on $E$ and $U: E \rightarrow E$ is a nonexpansive mapping on $E$. Obviously, $\left\{\beta_{n}\right\}$ satisfies the conditions (i) $\beta_{n} \rightarrow 0$; (ii) $\sum_{n=1}^{\infty} \beta_{n}=\infty$; and 
(iii) $\sum_{n=1}^{\infty}\left|\beta_{n+1}-\beta_{n}\right|<\infty$ or $\frac{\beta_{n}}{\beta_{n+1}} \rightarrow 1(n \rightarrow \infty)$. By Proposition 2.8 (ii), we conclude that $\left\{y_{n}\right\}$ converges strongly a fixed point of $z$ of $U=T Q$, which solves the variational inequality:

$$
\langle(I-g) z, j(y-z)\rangle \geq 0, \quad \forall y \in \operatorname{Fix}(U)
$$

In view of Lemma 2.1, we know that $z=T Q z=T z$, which implies that $z \in C$ and hence $Q z=z$, which yields $g(z)=\Phi(z)$.

Consequently, $\left\{y_{n}\right\}$, and hence $\left\{x_{n}\right\}$, converges strongly to $z \in \operatorname{Fix}(T)$ which solves the variational inequality (VI):

$$
\langle(I-\Phi) z, j(y-z)\rangle \geq 0, \quad \forall y \in \operatorname{Fix}(T)
$$

The proof is completed.

Remark 3.1 Theorem 3.1 improves and generalizes Theorem 2.2 due to Song and Chen [5]; Theorem 3.2 improves and generalizes Theorem 2.4 due to Song and Chen [5] and Theorem 4.2 due to Matsushita and Takahashi [6]. We point out that our method of argumentation is much simpler than the ones used by Song and Chen [5], and Matsushita and Takahashi [6].

In order to establish the strong convergence of (2.9), we first consider a special case of (2.9):

Fix $\lambda \in(0,1), x_{1} \in C, \forall u \in C, \quad x_{n+1}=\lambda x_{n}+(1-\lambda) Q\left[\alpha_{n} u+\left(1-\alpha_{n}\right) T x_{n}\right], \quad n \geq 1$.

Theorem 3.3 Let $C$ be a nonempty closed convex subset of a reflexive and strictly convex Banach space E, whose norm is uniformly Gâteaux differentiable. Let $T: C \rightarrow E$ be a nonexpansive mapping with $\operatorname{Fix}(T) \neq \emptyset$. Suppose also that $C$ is a sunny nonexpansive retract of $E$ and let $Q$ be the unique sunny nonexpansive retraction from $E$ onto $C$. Let $\left\{\alpha_{n}\right\}$ be a sequence satisfying conditions $(\mathrm{C} 1) \alpha_{n} \rightarrow 0$ and $(\mathrm{C} 2) \sum_{n=1}^{\infty} \alpha_{n}=\infty$. Then the sequence $\left\{x_{n}\right\}$ defined by (3.8) converges strongly to a fixed point $z$ of $T$, which solves the following variational inequality:

$$
\langle z-u, j(y-z)| \geq 0, \quad \forall y \in \operatorname{Fix}(T) .
$$

Proof We split the proof into four steps.

Step 1. $\left\{x_{n}\right\}$ is bounded.

Indeed, set $y_{n}=Q\left[\alpha_{n} u+\left(1-\alpha_{n}\right) T\left(x_{n}\right)\right]$, and take a fixed point $p \in \operatorname{Fix}(T)$. Then we have

$$
\begin{aligned}
\left\|x_{n+1}-p\right\| & \leq \lambda\left\|x_{n}-p\right\|+(1-\lambda)\left\|y_{n}-p\right\| \\
& \leq \lambda\left\|x_{n}-p\right\|+(1-\lambda)\left[\alpha_{n}\|u-p\|+\left(1-\alpha_{n}\right)\left\|x_{n}-p\right\|\right] \\
& =\left[1-(1-\lambda) \alpha_{n}\right]\left\|x_{n}-p\right\|+(1-\lambda) \alpha_{n}\|u-p\| \\
& \leq \max \left\{\left\|x_{1}-p\right\|,\|u-p\|\right\}=M
\end{aligned}
$$

for all $n \geq 1$, hence $\left\{x_{n}\right\}$ is bounded, so is $\left\{T x_{n}\right\}$. 
Step 2. $x_{n}-y_{n} \rightarrow 0(n \rightarrow \infty)$.

Observe that

$$
\begin{aligned}
\left\|y_{n+1}-y_{n}\right\| & =\left\|Q\left[\alpha_{n+1} u+\left(1-\alpha_{n+1}\right) T x_{n+1}\right]-Q\left[\alpha_{n} u+\left(1-\alpha_{n}\right) T x_{n}\right]\right\| \\
& \leq\left\|\left(\alpha_{n+1}-\alpha_{n}\right) u+\left(1-\alpha_{n+1}\right) T x_{n+1}-\left(1-\alpha_{n}\right) T x_{n}\right\| \\
& \leq\left\|x_{n+1}-x_{n}\right\|+\left|\alpha_{n+1}-\alpha_{n}\right|\left(\|u\|+\left\|T x_{n}\right\|\right) \\
& \leq\left\|x_{n+1}-x_{n}\right\|+M_{1}\left|\alpha_{n+1}-\alpha_{n}\right|
\end{aligned}
$$

for some positive constant $M_{1}$.

We have

$$
\varlimsup_{n}\left(\left\|y_{n+1}-y_{n}\right\|-\left\|x_{n+1}-x_{n}\right\|\right) \leq 0 .
$$

By virtue of Proposition 2.9, we claim that $x_{n}-y_{n} \rightarrow 0(n \rightarrow \infty)$.

Step 3. $\overline{\lim }_{n}\left\langle u-z, j\left(x_{n}-z\right)\right\rangle \leq 0$, where $z=Q_{\mathrm{Fix}(T)} u$, and $Q_{\mathrm{Fix}(T)}: C \rightarrow \operatorname{Fix}(T)$ is the unique sunny nonexpansive retraction from $C$ onto $\operatorname{Fix}(T)$, which is guaranteed by Theorem 3.1.

Notice that

$$
\begin{aligned}
\left\|y_{n}-Q T x_{n}\right\| & \leq \alpha_{n}\left(\|u\|+\left\|T x_{n}\right\|\right) \\
& \leq M_{1} \alpha_{n} \rightarrow 0,
\end{aligned}
$$

and $\left\|x_{n}-y_{n}\right\| \rightarrow 0$, we have

$$
\left\|x_{n}-Q T x_{n}\right\| \leq\left\|x_{n}-y_{n}\right\|+\left\|y_{n}-Q T x_{n}\right\| \rightarrow 0 .
$$

For $t \in(0,1)$, let $\left\{x_{t}\right\}$ be the path defined by

$$
x_{t}=t u+(1-t) Q T x_{t} .
$$

Since $Q T: C \rightarrow C$ is a nonexpansive self-mapping, using Theorem 2 of Morales and Jung [12], we see that $\left\{x_{t}\right\}$ converges strongly to $z \in \operatorname{Fix}(Q T)=\operatorname{Fix}(T)$ by Lemma 2.1 as $t \rightarrow 0$, where $z=Q_{\operatorname{Fix}(T)} u$. Using Lemma 2.1 of Song et al. [13], we conclude that

$$
\varlimsup_{n}\left\langle u-z, j\left(x_{n}-z\right)\right\rangle \leq 0 .
$$

Step 4. $x_{n} \rightarrow z(n \rightarrow \infty)$.

By using the definition of $\left\{y_{n}\right\}$ and Proposition 2.2(3), we have

$$
\begin{aligned}
\left\|y_{n}-z\right\|^{2} & =\left\|Q\left[\alpha_{n} u+\left(1-\alpha_{n}\right) T x_{n}\right]-Q z\right\|^{2} \\
& \leq\left\langle\alpha_{n}(u-z)+\left(1-\alpha_{n}\right)\left(T x_{n}-z\right), j\left(y_{n}-z\right)\right\rangle \\
& =\alpha_{n}\left\langle u-z, j\left(y_{n}-z\right)\right\rangle+\left(1-\alpha_{n}\right)\left\langle T x_{n}-z, j\left(y_{n}-z\right)\right\rangle \\
& \leq \alpha_{n}\left\langle u-z, j\left(y_{n}-z\right)\right\rangle+\frac{1-\alpha_{n}}{2}\left\|T x_{n}-z\right\|^{2}+\frac{1-\alpha_{n}}{2}\left\|y_{n}-z\right\|^{2} \\
& \leq \alpha_{n}\left\langle u-z, j\left(y_{n}-z\right)\right\rangle+\frac{1-\alpha_{n}}{2}\left\|x_{n}-z\right\|^{2}+\frac{1-\alpha_{n}}{2}\left\|y_{n}-z\right\|^{2},
\end{aligned}
$$


from which one derives

$$
\left\|y_{n}-z\right\|^{2} \leq\left(1-\alpha_{n}\right)\left\|x_{n}-z\right\|^{2}+2 \alpha_{n}\left\langle u-z, j\left(y_{n}-z\right)\right\rangle .
$$

By virtue of (3.8), (3.10), and the convexity of $\|\cdot\|^{2}$, we have

$$
\begin{aligned}
&\left\|x_{n+1}-z\right\|^{2} \\
& \leq \lambda\left\|x_{n}-z\right\|^{2}+(1-\lambda)\left\|y_{n}-z\right\|^{2} \\
& \leq \lambda\left\|x_{n}-z\right\|^{2}+(1-\lambda)\left(1-\alpha_{n}\right)\left\|x_{n}-z\right\|^{2}+2(1-\lambda) \alpha_{n}\left\langle u-z, j\left(y_{n}-z\right)\right\rangle \\
&= {\left[1-(1-\lambda) \alpha_{n}\right]\left\|x_{n}-z\right\|^{2}+2(1-\lambda) \alpha_{n}\left\langle u-z, j\left(y_{n}-z\right)\right\rangle } \\
&= {\left[1-(1-\lambda) \alpha_{n}\right]\left\|x_{n}-z\right\|^{2}+2(1-\lambda) \alpha_{n}\left\langle u-z, j\left(x_{n}-z\right)\right\rangle } \\
&+2(1-\lambda) \alpha_{n}\left\{u-z, j\left(y_{n}-z\right)-j\left(x_{n}-z\right)\right\rangle \\
& \leq {\left[1-(1-\lambda) \alpha_{n}\right]\left\|x_{n}-z\right\|^{2}+2(1-\lambda) \alpha_{n} \sigma_{n}+e_{n}, }
\end{aligned}
$$

where $\sigma_{n}=\max \left\{0,\left\langle u-z, j\left(x_{n}-z\right)\right\rangle\right\}$ and $e_{n}=2(1-\lambda) \alpha_{n}\left\langle u-z, j\left(y_{n}-z\right)-j\left(x_{n}-z\right)\right\rangle$. It is easily to see that $\sigma_{n} \geq 0$ and $\sigma_{n} \rightarrow 0(n \rightarrow \infty)$ in view of step 3 . Now we shall show that $e_{n}=o\left(\alpha_{n}\right)$. Indeed, from step 1 , we know that $\left\{x_{n}-z\right\}$ and $\left\{y_{n}-z\right\}$ are bounded. By step 2 , $y_{n}-x_{n}=\left(y_{n}-z\right)-\left(x_{n}-x\right) \rightarrow 0$, it follows from Proposition 2.1(4) that

$$
\left\langle u-z, j\left(y_{n}-z\right)-j\left(x_{n}-z\right)\right\rangle \rightarrow 0,
$$

and hence $e_{n}=o\left(\alpha_{n}\right)$. Consequently, (3.11) reduces to

$$
\left\|x_{n+1}-z\right\|^{2} \leq\left[1-(1-\lambda) \alpha_{n}\right]\left\|x_{n}-z\right\|^{2}+o\left(\alpha_{n}\right) .
$$

Set $a_{n}=\left\|x_{n}-z\right\|^{2}, t_{n}=(1-\lambda) \alpha_{n}$. Then (3.12) reduces to

$$
a_{n+1} \leq\left(1-t_{n}\right) a_{n}+o\left(t_{n}\right)
$$

where $\left\{t_{n}\right\}$ satisfies condition (i) $t_{n} \rightarrow 0$; and (ii) $\sum_{n=1}^{\infty} t_{n}=\infty$. By Proposition 2.10 , we conclude that $a_{n} \rightarrow 0(n \rightarrow \infty)$, i.e., $x_{n} \rightarrow z(n \rightarrow \infty)$. This completes the proof.

Now we prove the strong convergence of (2.9).

Theorem 3.4 Let $E$ be a real reflexive and strictly convex Banach space whose norm is uniformly Gâteaux differentiable, let $C$ be a nonempty closed convex subset of $E$, let $T$ be a nonexpansive mapping from $C$ into $E$ with $\operatorname{Fix}(T) \neq \emptyset$ and let $\Phi: C \rightarrow C$ be a MKC. Suppose that $C$ is a sunny nonexpansive retract of $E$. Let $\left\{\alpha_{n}\right\}$ be a real sequence such that $0 \leq \alpha_{n} \leq 1, \alpha_{n} \rightarrow 0$ and $\sum_{n=1}^{\infty} \alpha_{n}=\infty$. Let $\lambda \in(0,1)$ and $x_{1} \in C$. Suppose that $\left\{x_{n}\right\}$ is given by (2.9). Then $\left\{x_{n}\right\}$ converges strongly to $z \in \operatorname{Fix}(T)$ which solves the variational inequality (VI):

$$
\langle(I-\Phi) z, j(y-z)\rangle \geq 0, \quad \forall y \in \operatorname{Fix}(T) .
$$


Proof By Proposition 2.7(i), we know that $Q_{\mathrm{Fix}(T)} \Phi: C \rightarrow C$ is a MKC on $C$. It follows from Theorem MK, $Q_{\mathrm{Fix}(T)} \Phi$ has a unique fixed point $z$ in $C$, i.e., $z=Q_{\mathrm{Fix}(T)} \Phi(z)$.

Let $y_{1} \in C$ and define a sequence $\left\{y_{n}\right\}$ by

$$
y_{n+1}=\lambda y_{n}+(1-\lambda) Q\left[\alpha_{n} \Phi(z)+\left(1-\alpha_{n}\right) T y_{n}\right], \quad n \geq 1 .
$$

From Theorem 3.3, we assert that

$$
y_{n} \rightarrow z=Q_{\operatorname{Fix}(T)} \Phi(z)
$$

where $z \in \operatorname{Fix}(T)$ which solves the variational inequality (VI):

$$
\langle(I-\Phi) z, j(y-z)| \geq 0, \quad \forall y \in \operatorname{Fix}(T) .
$$

We shall prove that $x_{n}-y_{n} \rightarrow 0$ as $n \rightarrow \infty$.

Assume that $a=\varlimsup_{n}\left\|x_{n}-y_{n}\right\|>0$; then $\forall \varepsilon>0, \varepsilon<a$, we can choose $\eta>0$ such that

$$
\varlimsup_{n}\left\|x_{n}-y_{n}\right\|>\varepsilon+\eta
$$

For above $\varepsilon>0$, using Proposition 2.6, we know that there exists $\gamma \in(0,1)$ such that

$$
\|\Phi x-\Phi y\| \leq \gamma\|x-y\|
$$

for all $x, y \in C$ with $\|x-y\| \geq \varepsilon$, which implies that

$$
\|\Phi x-\Phi y\| \leq \max \{\gamma\|x-y\|, \varepsilon\}
$$

for all $x, y \in C$.

Since $y_{n} \rightarrow z$ as $n \rightarrow \infty$, we see that there exists some $n_{0} \geq 1$ such that

$$
\left\|y_{n}-z\right\| \leq(1-\gamma) \eta
$$

for all $n \geq n_{0}$.

We now consider two possible cases.

Case 1. There exists some $v_{1} \geq n_{0}$ such that

$$
\left\|x_{v_{1}}-y_{v_{1}}\right\| \leq \varepsilon+\eta
$$

By using (2.9), (3.13), (3.16), and (3.17), we have

$$
\begin{aligned}
& \left\|x_{v_{1}+1}-y_{v_{1}+1}\right\| \\
& \leq \lambda\left\|x_{v_{1}}-y_{v_{1}}\right\|+(1-\lambda)\left[\alpha_{v_{1}}\left\|\Phi x_{v_{1}}-\Phi z\right\|+\left(1-\alpha_{v_{1}}\right)\left\|x_{v_{1}}-y_{v_{1}}\right\|\right] \\
& =\left[1-(1-\lambda) \alpha_{v_{1}}\right]\left\|x_{v_{1}}-y_{v_{1}}\right\|+(1-\lambda) \alpha_{v_{1}}\left\|\Phi x_{v_{1}}-\Phi z\right\| \\
& \leq\left[1-(1-\lambda) \alpha_{v_{1}}\right]\left\|x_{v_{1}}-y_{v_{1}}\right\|+(1-\lambda) \alpha_{v_{1}}\left\|\Phi x_{v_{1}}-\Phi y_{v_{1}}\right\| \\
& \quad+(1-\lambda) \alpha_{v_{1}}\left\|\Phi y_{v_{1}}-\Phi z\right\|
\end{aligned}
$$




$$
\begin{aligned}
\leq & {\left[1-(1-\lambda) \alpha_{v_{1}}\right]\left\|x_{v_{1}}-y_{v_{1}}\right\|+(1-\lambda) \alpha_{v_{1}} \max \left\{\gamma\left\|x_{v_{1}}-y_{v_{1}}\right\|, \varepsilon\right\} } \\
& +(1-\lambda) \alpha_{v_{1}}\left\|y_{v_{1}}-z\right\| \\
\leq & \max \left\{\left[1-(1-\gamma)(1-\lambda) \alpha_{v_{1}}\right] \varepsilon+(1-\gamma)(1-\lambda) \alpha_{v_{1}} \eta\right. \\
& {\left.\left[1-(1-\lambda) \alpha_{v_{1}}\right] \varepsilon+(1-\lambda) \alpha_{v_{1}}(\varepsilon+\eta)\right\} } \\
\leq & \varepsilon+\eta .
\end{aligned}
$$

Similarly, we have

$$
\left\|x_{v_{1}+2}-y_{v_{1}+2}\right\| \leq \varepsilon+\eta \text {. }
$$

By induction, we have

$$
\left\|x_{v_{1}+m}-y_{v_{1}+m}\right\| \leq \varepsilon+\eta
$$

for all $m \geq 1$, which implies that

$$
\varlimsup_{n}\left\|x_{n}-y_{n}\right\| \leq \varepsilon+\eta
$$

which contradicts with (3.14). Consequently, $x_{n}-y_{n} \rightarrow 0$, and hence $x_{n} \rightarrow z \in \operatorname{Fix}(T)$ which solves the variational inequality (VI).

Case 2. $\left\|x_{n}-y_{n}\right\|>\varepsilon+\eta$ for all $n \geq v_{1}$.

We shall prove that case 2 is impossible. Suppose case 2 holds true. By (3.15), we have

$$
\left\|\Phi x_{n}-\Phi y_{n}\right\| \leq \gamma\left\|x_{n}-y_{n}\right\|
$$

for all $n \geq v_{1}$.

By using (2.9), (3.13), and (3.19), we have

$$
\begin{aligned}
& \left\|x_{n+1}-y_{n+1}\right\| \\
& \quad \leq \lambda\left\|x_{n}-y_{n}\right\|+(1-\lambda)\left[\alpha_{n}\left\|\Phi x_{n}-\Phi z\right\|+\left(1-\alpha_{n}\right)\left\|x_{n}-y_{n}\right\|\right] \\
& \quad \leq\left[1-(1-\lambda) \alpha_{n}\right]\left\|x_{n}-y_{n}\right\|+(1-\lambda) \alpha_{n}\left\|\Phi x_{n}-\Phi y_{n}\right\|+(1-\lambda) \alpha_{n}\left\|y_{n}-z\right\| \\
& \quad \leq\left[1-(1-\gamma)(1-\lambda) \alpha_{n}\right]\left\|x_{n}-y_{n}\right\|+o\left(\alpha_{n}\right),
\end{aligned}
$$

from which one derives

$$
x_{n}-y_{n} \rightarrow 0,
$$

in view of Proposition 2.10, and hence $0 \geq \varepsilon+\eta$, a contradiction, thus, case 2 is impossible. The proof is completed.

Remark 3.2 We do not know whether the conclusion of Theorem 3.4 holds true when $\Phi: C \rightarrow E$ is a nonself-MKC. However, in the case where $E$ is a Hilbert space, it is true. 
Applying Theorems 3.1-3.4 to a Hilbert space $H$, we can obtain new strong convergence theorems, which improve and generalize the corresponding results by Matsushita and Kuroiwa [14], and Zhou et al. [15].

Theorem 3.5 Let $H$ be a real Hilbert space, let $C$ be a nonempty closed convex subset of $H$, let $T$ be a nonexpansive mapping from $C$ into $H$ with $\operatorname{Fix}(T) \neq \emptyset$ and let $\Phi: C \rightarrow H$ be a MKC. Then the conclusions of Theorems 3.1-3.4 hold true.

\section{Concluding remarks}

This work contains our contribution dedicated to developing and improving the viscosity approximation methods for finding fixed points of nonexpansive nonself-mappings. A novel and remarkable finding is contained in Lemma 2.1, that is, $\operatorname{Fix}(T)=\operatorname{Fix}(T Q)$, which makes it possible to invent a novel and simple method of argumentation for establishing strong convergence theorems. We have introduced our modified viscosity approximation methods for finding fixed points of nonexpansive nonself-mappings. Applying our main results to a Hilbert space, we have drawn the corresponding conclusions announced by some authors.

\section{Competing interests}

The authors declare that they have no competing interests.

\section{Authors' contributions}

Both authors have played equal roles in deriving results and writing of this paper. Both authors read and approved the final manuscript.

\section{Acknowledgements}

The first author was supported in part by NSFC 11071053. The authors wish to thank the referee for the valuable suggestions to improve the writing of this paper.

Received: 12 August 2013 Accepted: 25 February 2014 Published: 12 Mar 2014

\section{References}

1. Ceng, LC, Xu, HK, Yao, JC: Strong convergence of an iterative method with perturbed mappings for nonexpansive and accretive operators. Numer. Funct. Anal. Optim. 29, 1-22 (2008)

2. Takahashi, W, Yao, JC, Kocourek, P: Weak and strong convergence theorems for generalized hybrid nonself-mappings in Hilbert spaces. J. Nonlinear Convex Anal. 11, 567-586 (2010)

3. Naraghirad, E, Takahashi, W, Yao, JC: Generalized retraction and fixed point theorems using Bregman functions in Banach spaces. J. Nonlinear Convex Anal. 13, 141-156 (2012)

4. Takahashi, W, Wong, NC, Yao, JC: Fixed point theorems for nonlinear non-self mappings in Hilbert spaces and applications. Fixed Point Theory Appl. 2013, 116 (2013)

5. Song, Y, Chen, R: Viscosity approximation methods for nonexpansive nonself-mappings. J. Math. Anal. Appl. 321 , 316-326 (2006)

6. Matsushita, S, Takahashi, W: Strong convergence theorems for nonexpansive nonself-mappings without boundary conditions. Nonlinear Anal. 68, 412-419 (2008)

7. Cioranescu, I: Geometry of Banach Spaces, Duality Mappings and Nonlinear Problems. Kluwer Academic, Dordrecht (1990)

8. Takahashi, W: Nonlinear Functional Analysis. Yokohama Publishers, Yokohama (2000)

9. Matsushita, S, Kuroiwa, D: Approximation of fixed points of nonexpansive nonself-mappings. Sci. Math. Jpn. 57, $171-176(2003)$

10. Meir, A, Keeler, E: A theorem on contractions. J. Math. Anal. Appl. 28, 326-329 (1969)

11. Suzuki, T: Moudafi's viscosity approximations with Meir-Keeler contractions. J. Math. Anal. Appl. 325, 342-352 (2007)

12. Morales, C, Jung, J: Convergence of paths for pseudo-contractive mappings in Banach spaces. Proc. Am. Math. Soc. $128,3411-3419(2000)$

13. Song, $\mathrm{Y}, \mathrm{Chen}, \mathrm{R}$, Zhou, $\mathrm{H}$ : Viscosity approximation methods for nonexpansive mapping sequences in Banach spaces. Nonlinear Anal. 66, 1016-1024 (2007)

14. Matsushita, S, Kuroiwa, D: Strong convergence of averaging iterations of nonexpansive nonself-mappings. J. Math. Anal. Appl. 294, 206-214 (2004)

15. Zhou, HY, Wang, PY, Zhou, Y: Minimum-norm fixed point of nonexpansive mappings with applications. Optimization, 1-16 (2013). doi:10.1080/02331934.2013.811667 